\title{
Analysis of Retinal Nerve Fibre Layer and Optic Nerve Head by Optical Coherence Tomography in POAG
}

\section{CHINMAYEE PABOLU, KRISHNAGOPAL SRIKANTH, KIRTI NATH JHA, A R RAJALAKSHMI, NAGARAJAN SWATHI}

\section{ABSTRACT}

Introduction: Glaucoma is the second leading cause of blindness. Early identification of disease is important, as treatment can slow the disease progression and preserve vision.

Aim: The purpose of our study was to evaluate the Retinal Nerve Fibre Layer (RNFL) and Optic nerve head (ONH) using Optical Coherence Tomography (OCT) in primary open angle glaucoma (POAG) and to determine if any correlation exists between the two.

Materials and Methods: In a Cross-sectional study including, 73 eyes of 39 patients with POAG, aged 40 years and above, we evaluated retinal nerve fiber layer (RNFL) and optic nerve head (ONH) changes using optical coherence tomography (OCT). The statistical analysis was done using Epi Info software.

Results: The mean age was $62.82 \pm 9.78$ years and
IOP was $24.25 \pm 3.685 \mathrm{mmHg}$. Mean thickness of RNFL was $101.67 \pm 25.8 \mu \mathrm{m}, 60.21 \pm 14.02 \mu \mathrm{m}, 98.67 \pm 25.10 \mu \mathrm{m}$, $72.44 \pm 21.914 \mu \mathrm{m}$ of superior, temporal, inferior and nasal quadrants respectively. The $\mathrm{ONH}$ parameters and the RNFL thickness were quantified and analyzed. It was found that most of the patients had more thinning in the superior and inferior quadrants. Superior RNFL and the vertical ONH parameters showed best correlation (-0.439, p-value 0.005).

Conclusion: SD-OCT is a powerful objective and structural assessment tool in diagnosing and managing glaucoma and hence should be a part of routine glaucoma evaluation. Correlation of ONH topography with RNFL thickness may be of value in patients with suspicious disc picture. Superior quadrant of the RNFL was found to be the most reliable parameter in primary open angle glaucoma.

Keywords: Cup disc ratio, Disc area, Glaucoma, Peripapillary nerve fiber thickness

\section{INTRODUCTION}

Glaucoma is an optic neuropathy characterized by progressive loss of retinal ganglion cells and optic nerve damage resulting in visual field loss and eventual blindness [1]. It is the second leading cause of blindness worldwide. In India the burden is estimated about 11.2 million in adult population [2].

Early diagnosis and appropriate intervention can delay the natural history of the disease. Retinal function can be measured with psychophysical techniques such as standard automated perimetry, short-wavelength automated perimetry, and frequency-doubling technology, but more than $1 / 3^{\text {rd }}$ of axonal damage would have already occurred before it is detected by perimetry [3].

A tool that detects axonal damage before functional deficit is manifested (i.e. pre-perimetric damage) is of immense value in glaucoma. Spectral Domain Optical Coherence Tomography (SD-OCT) based on Michaelson interferometry provides high-resolution images of the retinal nerve fiber layer (RNFL) [4]. The images of the RNFL and optic nerve head $(\mathrm{ONH})$ obtained by OCT are proving to be invaluable in diagnosis and management of primary open angle glaucoma (POAG).

However, there is as yet no clear consensus on the correlation between $\mathrm{ONH}$ and RNFL, both of which are affected in the disease [5]. We have evaluated the RNFL and $\mathrm{ONH}$ parameters in known POAG patients to determine possible correlation between them.

\section{MATERIALS AND METHODS}

This cross-sectional study was conducted in Mahatma Gandhi Medical College and Research Institute, Pondicherry, from January 2014 to June 2015 for a period of 18 months. Institutional human ethical committee approval was obtained. All patients diagnosed as POAG, above the age of 40 years were included in the study. Patients with uveitis, 
secondary glaucoma, high myopia, retinal pathologies and media opacities causing difficulty in performing the OCT were excluded from the study. Following routine clinical examination, RNFL and $\mathrm{ONH}$ images were obtained on Optos $^{\circledR}$ SD-OCT (Spectral Domain - Optical Coherence Tomography).

RNFL thickness was measured with a $3.4 \mathrm{~mm}$ diameter rim placed around the optic nerve. The software tracks the position of the RNFL OCT scan and its position in relation to the optic nerve. The RNFL thickness map is displayed along with colour coding for easy understanding. For $\mathrm{ONH}$ analysis, optic nerve topography mode was used. A 3D tomographic image of the optic nerve was generated from a stack of sequential OCT and confocal SLO images. An in-built software analyzed the data in the machine and a report was created on: disc area, cup area, rim area, cup/ disc area, mean cup depth and maximum cup depth. The measurements were compared to set parameters in the normative database. To be acceptable for inclusion the signal strength was taken into consideration. OCT scans, with signal strength (signal noise ratio - SNR) of $>6$ were included.The statistical analysis was done using Epi Info software. Mean and standard deviation (SD) values with 95\% confidence intervals were calculated for all RNFL and $\mathrm{ONH}$ parameters. Pearson's correlation was applied to determine correlation between RNFL thickness and $\mathrm{ONH}$ parameters. $p$-value of $<0.05$ was considered statistically significant for the study.

\section{RESULTS}

73 eyes of 39 patients ( 23 males and 16 females) above 40 years of age with POAG were included in the study. The mean age was $62.82 \pm 9.78$ years. The mean IOP was $24.25 \pm 3.69 \mathrm{~mm}$ of $\mathrm{Hg}$ and the mean CCT was $566 \pm 2.1 \mu$ and SNR was 6.8 .

RNFL thickness of all the patients were analyzed for each quadrant separately. Most of the patients had more thinning in the superior and inferior quadrants [Table/Fig-1]. $\mathrm{ONH}$ parameters include disc area, cup area, rim area, horizontal $C: D$, vertical C:D, C:D area ratio, mean cup depth and maximum cup depth [Table/Fig-2]. The mean RNFL thickness of all the quadrants showed a statistically significant correlation with the $\mathrm{ONH}$ parameters of which superior quadrant showing the best correlation [Table/Fig-3 and 4].

\begin{tabular}{l|c|c|}
\hline Parameter & Mean \pm SD & $95 \% \mathrm{Cl}$ \\
\hline SUP & $101.67 \pm 25.81 \mu$ & $95.65-107.69$ \\
\hline TEM & $60.21 \pm 14.02 \mu$ & $56.93-63.48$ \\
\hline INF & $98.67 \pm 25.10 \mu$ & $92.81-104.53$ \\
\hline NAS & $72.44 \pm 21.91 \mu$ & $67.33-77.55$ \\
\hline \\
[Table/Fig-1]: RNFL Analysis. \\
*SUP -Superior, TEM - Temporal, INF - Inferior, NAS- Nasal, Cl - Confidence Interval.
\end{tabular}

\begin{tabular}{|l|c|c|}
\hline Parameter & Mean \pm SD & $95 \% \mathrm{Cl}$ \\
\hline Disc Area $\left(\mathrm{mm}^{2}\right)$ & $3.153 \pm 1.171$ & $2.872-3.421$ \\
\hline Cup Area $\left(\mathrm{mm}^{2}\right)$ & $2.312 \pm 1.102$ & $2.062-2.573$ \\
\hline Rim Area $\left(\mathrm{mm}^{2}\right)$ & $0.890 \pm 0.817$ & $0.699-1.081$ \\
\hline C:D Horizontal & $0.892 \pm 0.157$ & $0.855-0.929$ \\
\hline C:D Vertical & $0.857 \pm 0.147$ & $0.822-0.890$ \\
\hline C:D Area Ratio & $0.724 \pm 0.241$ & $0.668-0.780$ \\
\hline Mean Cup Depth $(\mathrm{mm})$ & $0.256 \pm 0.142$ & $0.263-0.329$ \\
\hline Max Cup Depth $(\mathrm{mm})$ & $0.640 \pm 0.201$ & $0.593-0.687$
\end{tabular}

[Table/Fig-2]: ONH analysis.

"SUP -Superior, TEM - Temporal, INF - Inferior, NAS- Nasal, CI - Confidence Interval.

\begin{tabular}{|l|l|}
\hline RNFL & C : D VERTICAL \\
\hline Superior & $r=-0.43 p-$ value -0.005 \\
\hline Inferior & $r=-0.385 p-$ value -0.001 \\
\hline
\end{tabular}

[Table/Fig-3]: Correlation between the superior and inferio quadrants of RNFL with the vertical C:D.

* RNFL - Retinal nerve fiber layer, SUP - Superior, INF - Inferior, C:D - Cup Disc ratio.

\begin{tabular}{|l|l|}
\hline RNFL & C:D HORIZONTAL \\
\hline Nasal & $r=-0.31 p-$ value $=0.006$ \\
\hline Temporal & $r=-0.21 p$ - value -0.036 \\
\hline
\end{tabular}

[Table/Fig-4]: Correlation between the nasal and temporal quadrants of RNFL with the horizontal C:D.

*RNFL - Retinal nerve fiber layer, NAS - Nasal, TEM - Temporal, C:D - Cup Disc ratio.

\section{DISCUSSION}

OCT has now become almost a mandatory tool for diagnosis and follow-up of POAG. The instrument measures various parameters in the optic disc and also compares the patient's values with normative database available. Traditional description of increased cup disc ratio (C:D), vertically oval cup favors $\mathrm{ONH}$ topography as an important parameter. Ganglion cell loss, the primary event in glaucomatous damage is better reflected in RNFL thickness [6]. There is a wide normative variation in $\mathrm{ONH}$ topography [7]. This coupled with observations of RNFL thinning prior to perimetric defects argues in favor of RNFL thickness as the better suited parameter in glaucoma detection [8].

RNFL thickness is measured by a circular scan of $3.4 \mathrm{~mm}$ diameter placed concentric to the disc. Because this is a fixed size ring, the distance from the disc where RNFL thickness is measured would vary with disc diameter [8]. Further studies revealed an increased optic nerve fiber count in larger size discs [9]. A smaller disc size would register a thinner RNFL either due to anatomically lesser optic nerve fiber count or due to greater distance of measurement from the disc, increases the chances of being falsely diagnosed as glaucoma. Similarly, in a larger disc, with thicker RNFL, early loss is likely to be missed as this would still be within the normal curve. Also age related thinning of RNFL in the presence of normal $\mathrm{ONH}$ morphology cautions against diagnosis of POAG based only on RNFL curve [5]. 
Disc area ranging from 1.8 to $5.3 \mathrm{~mm}^{2}$; we used a fixed ring size in proven glaucomatous eyes. Mansoori et al., observed an inverse correlation between RNFL thickness and C:D in all quadrants in a study of normal eyes [10]. In this study of glaucomatous eyes we observed a similar relationship, which was most for superior RNFL thickness and vertical C:D, similar to a study done by Khanal et al., [11]. Few previous studies suggest that, thinning in the inferior quadrant being more sensitive measure in open angle glaucoma [12-14]. In our study both the inferior and superior quadrants correlated well with the vertical $\mathrm{C}: \mathrm{D}$, but superior being the most sensitive.

Though measuring the RNFL thickness provides information regarding the extent of glaucomatous damage, it has to be correlated clinically for deciding the best management. Hence, SD- OCT plays a key role in the management of open angle glaucoma.

\section{LIMITATION}

A larger sample size would have been of value in determining the role of age as a confounding factor in RNFL thickness.

\section{CONCLUSION}

SD-OCT is a powerful non-invasive objective and structural assessment tool in diagnosing and managing glaucoma hence should be a part of routine glaucoma evaluation. It is not prudent to diagnose glaucoma by RNFL thickness curve. It is inarguably an excellent tool in follow up of glaucoma progression. Correlation of $\mathrm{ONH}$ topography with superior RNFL thickness may be of value in patients with suspicious disc picture. Superior quadrant of the RNFL was found to be the most reliable parameter in primary open angle glaucoma.

\section{REFERENCES}

[1] American Academy of Ophthalmology. Preferred Practice Pattern: Primary Open-Angle Glaucoma. 2003.

[2] George R, Ve RS, Vijaya L. Glaucoma in India: Estimated burden of Disease: J Glaucoma. 2010;19(6):391-97.

[3] Tatham AJ, Weinreb RN, Medeiros FA. Strategies for improving early detection of glaucoma: the combined structure \& function index. Clin Ophthalmol. 2014;8;611-21.

[4] Jaffe GJ, Caprioli J. Optical coherence tomography to detect and manage retinal disease and glaucoma. Am J Ophthalmol. 2004;137(1):156-69.

[5] Mansoori T, Viswanath K, Balakrishna N. Correlation between peripapillary retinal nerve fiber layer thickness and optic nerve head parameters using spectral domain optical coherence tomography. J Glaucoma. 2010;19(9):604-08

[6] Blumenthal EZ, Weinreb RN. Assessment of the retinal nerve fiber layer in clinical trials of glaucoma neuroprotection. Surv Ophthalmol. 2001;45(Suppl 3):S305-12; discussion S332-34.

[7] Tariq YM, Li H, Burlutsky G, Mitchell P. Retinal nerve fiber layer and optic disc measurements by spectral domain OCT: normative values and associations in young adults. Eye. 2012;26(12):1563-70.

[8] Savini G. Correlation between retinal nerve fibre layer thickness and optic nerve head size: an optical coherence tomography study. $\mathrm{Br} J$ Ophthalmol. 2005;89(4):489-92.

[9] Jonas JB, Budde WM. Diagnosis and pathogenesis of glaucomatous optic neuropathy: morphological aspects. Prog Retin Eye Res. 2000;19(1):1-40.

[10] Mansoori T, Viswanath K, Balakrishna N. Quantification of retinal nerve fiber layer thickness using spectral domain optical coherence tomography in normal Indian population. Indian J Ophthalmol. 2012;60(6):555.

[11] Khanal S, Thapa M, Racette L, Johnson R, Davey PG, Joshi MR, et al. Retinal nerve fiber layer thickness in glaucomatous Nepalese eyes and its relation with visual field sensitivity. J Optom. 2014;7(4):217-24.

[12] Wollstein G, Schuman JS, Price LL, Aydin A, Beaton SA, Stark PC, et al. Optical coherence tomography (OCT) macular and peripapillary retinal nerve fiber layer measurements and automated visual fields. Am J Ophthalmol. 2004;138(2):218-25.

[13] Wollstein G, Ishikawa H, Wang J, Beaton SA, Schuman JS. Comparison of three optical coherence tomography scanning areas for detection of glaucomatous damage. Am J Ophthalmol. 2005;139(1):39-43.

[14] Medeiros FA, Zangwill LM, Bowd C, Vessani RM, Susanna R, Weinreb $\mathrm{RN}$. Evaluation of retinal nerve fiber layer, optic nerve head, and macular thickness measurements for glaucoma detection using optical coherence tomography. Am J Ophthalmol. 2005;139(1):44-55.
AUTHOR(S):
1. Dr. Chinmayee Pabolu
2. Dr. Krishnagopal Srikanth
3. Dr. Kirti Nath Jha
4. Dr. A R Rajalakshmi
5. Dr. Nagarajan Swathi

\section{PARTICULARS OF CONTRIBUTORS:}

1. Resident, Department of Ophthalmology, Mahatma Gandhi Medical College and Research Institute, Sri Balaji Vidyapeeth University, Puducherry, India.

2. Professor and HOD, Department of Ophthalmology, Mahatma Gandhi Medical College and Research Institute, Sri Balaji Vidyapeeth University, Puducherry, India.

3. Professor, Department of Ophthalmology, Mahatma Gandhi Medical College and Research Institute, Sri Balaji Vidyapeeth University, Puducherry, India.
4. Associate Professor, Department of Ophthalmology, Mahatma Gandhi Medical College and Research Institute, Sri Balaji Vidyapeeth University, Puducherry, India.

5. Associate Professor, Department of Ophthalmology, Mahatma Gandhi Medical College and Research Institute, Sri Balaji Vidyapeeth University, Puducherry, India.

\section{NAME, ADDRESS, E-MAIL ID OF THE CORRESPONDING AUTHOR:}

Dr. Krishnagopal Srikanth,

Department of Ophthalmology, Mahatma Gandhi Medical College and Research Institute, Pondicherry Cuddalore EC Road, Pillayarkuppam, Puducherry-607402, India.

E-mail: Kskanth99@rediffmail.com

FINANCIAL OR OTHER COMPETING INTERESTS: None.

Date of Publishing: Apr 01, 2016 